\title{
A three-dimensional nonlinear meshfree algorithm for simulating mechanical responses of soft tissue
}

\author{
G. Y. Zhang*, A. Wittek, G. R. Joldes, X. Jin and K. Miller \\ Intelligent Systems for Medicine Laboratory, School of Mechanical and Chemical Engineering, \\ The University of Western Australia, 35 Stirling Highway, Crawley/Perth WA 6009, Australia \\ *Corresponding author: Guiyong.zhang@uwa.edu.au
}

\begin{abstract}
This paper presents an effective three-dimensional (3D) nonlinear explicit dynamic meshfree algorithm for the simulation of soft tissue mechanical responses. In the algorithm soft tissues are considered to be hyperelastic and nearly incompressible materials. The algorithm is based on the element-free Galerkin (EFG) method using total Lagrangian formulation and moving least square (MLS) approximation. This approximation uses a relatively large number of nodes for shape functions creation, which can significantly delay mesh distortion in large deformation computations. Essential boundary conditions are imposed exactly by coupling MLS shape functions with a finite element (FE) interpolation in the close region of essential boundary. Although volumetric integration is not exact, the large support domains of the MLS shape functions alleviate some of the key weaknesses of FE methods such as hour-glassing and volumetric locking. Explicit integration is performed in time domain, using a recently proposed method to calculate the critical time step. Verification against the results obtained using the established nonlinear finite element procedures available in the ABAQUS code confirms the accuracy of the presented meshfree algorithm. Application of the algorithm in modeling of the brain indentation indicates its ability to facilitate robust and accurate prediction of the organ responses subjected to large localized deformations consistent with the loading due to surgery.
\end{abstract}

Keywords: Meshfree methods, nonlinear dynamics analysis, soft tissue

\section{Introduction}

Surgical simulators present a safe and potentially effective way for surgical training, and can also be used in robot and computer assisted surgery for operation planning. Robust, fast and accurate 
numerical algorithms for predicting soft tissue deformations have been identified as the key technology for the development of practical surgical simulators [1]. In the context of computational biomechanics, finite element (FE) algorithms accounting for both geometric (i.e. large deformations and strains) and material nonlinearities have been developed [2-4]. Although the literature indicates that the models implemented using FE algorithms can facilitate accurate prediction of the organ responses during surgery [5-7], they are still not widely adopted in surgical practice, mainly because of the difficulties in handling very large deformations and discontinuities due to surgical cutting.

With the latest developments, meshfree/meshless methods [8-10] have been suggested as a possible alternative to solve the above difficulties. A key feature of meshfree methods is that a predefined mesh is not necessary for field variable approximation/interpolation. Various meshfree algorithms have shown very good performance in handling mesh distortion for nonlinear problems [11-19]. However, there are still some open topics for nonlinear explicit dynamic analysis using meshfree methods, such as handling essential boundary conditions effectively, conducting numerical integration, and stable time step calculation. For the above reasons, only a few published works can be found on applying meshfree methods for nonlinear analysis of soft tissue simulation [20-24]. This work expands the meshfree total Lagrangian explicit dynamics (MTLED) algorithm presented in [20] through the use of coupled MLS and FE shape functions to accurately impose the essential boundary conditions, a more accurate volumetric integration, and the use of a new stable time step estimation method developed particularly for the explicit time integration in meshfree methods. These numerical techniques have been proposed elsewhere, and in this work we integrated them in a consistent verified meshfree algorithm for soft tissue modeling.

In the next section we briefly introduce the formulations of total Lagrangian explicit dynamics meshfree algorithm, and then present three important implementation issues: numerical integration, treatment of essential boundary conditions and calculating stable time step. Verification results are presented next, followed by the example of application in modeling of the body organs (brain indentation), discussions and conclusions.

\section{Meshfree Total Lagrangian Explicit Dynamics Algorithm}

The presented algorithm is based on the meshfree EFG method using the total Lagrangian formulation, where stresses and strains are measured with respect to the original configuration allowing for pre-computing of most spatial derivatives before the time-stepping procedure. The stress (second Piola-Kirchoff stress) and strain (Green strain) measures used are appropriate for the 
treatment of large deformations. Explicit time integration eliminates the need for solving large systems of equations during the time-stepping procedure.

\subsection{Moving Least Square Approximation}

Details of the MLS shape functions construction are available in the literatures [25, 26]. The discrete MLS approximation of a function $u(\mathbf{X})$, denoted by $u^{h}(\mathbf{X})$, is constructed by a combination of monomials using $n$ nodal parameters as

$u^{h}(\mathbf{X})=\sum_{i=1}^{m} p_{i}(\mathbf{X}) a_{i}(\mathbf{X})=\mathbf{P}^{T}(\mathbf{X}) \mathbf{a}(\mathbf{X})$

where $m$ is the number of terms of monomials (polynomial basis), $p_{i}(\mathbf{X})$ are basis functions, $\mathbf{X}$ is the position of a material point in the initial configuration, and $a_{i}(\mathbf{X})$ are the coefficients of the approximation.

By introducing a weight function $W\left(\mathbf{X}-\mathbf{X}_{I}\right)$ and minimizing the weighted L2 norm $\left(J^{M L S}\right)$ as

$$
J^{M L S}=\sum_{I=1}^{n} W\left(\mathbf{X}-\mathbf{X}_{I}\right)\left[u^{h}\left(\mathbf{X}, \mathbf{X}_{I}\right)-u\left(\mathbf{X}_{I}\right)\right]^{2}
$$

where $n$ is the number of nodes within the support of $\mathbf{X}$. Finally by calculating the coefficients through the minimization, the MLS approximation is obtained by

$$
u^{h}(\mathbf{X})=\sum_{I=1}^{n} \phi_{I}(\mathbf{X}) u_{I}=\mathbf{\Phi}(\mathbf{X}) \mathbf{U}_{s}
$$

where $\mathbf{U}_{s}$ is the vector collecting the nodal parameters of the field variables for all the nodes in the local support domain, and $\phi_{I}(\mathbf{X})$ is the MLS shape function.

In this work, we create the MLS shape functions by using a linear polynomial basis and quartic spline weight functions, defined as

$$
W\left(\mathbf{X}-\mathbf{X}_{I}\right)=\left\{\begin{array}{cc}
1-6 \bar{d}^{2}+8 \bar{d}^{3}-3 \bar{d}^{4} & \text { for } \bar{d} \leq 1 \\
0 & \text { for } \bar{d}>1
\end{array}, \quad \bar{d}=\frac{\left|\mathbf{X}-\mathbf{X}_{I}\right|}{d_{w I}}\right.
$$

where $d_{w I}$ is the radius of the spherical influence domain of node $\mathbf{X}_{I}$. We calculate $d_{w I}$ using the average distance between node $\mathbf{X}_{I}$ and its neighboring nodes, multiplied by a dilatation parameter. This technique leads to high quality MLS shape functions even for irregularly distributed field nodes. 


\subsection{Total Lagrangian Explicit Dynamics Algorithm}

After introducing MLS approximation into the weak form of governing equations of solid mechanics using the total Lagrangian formulation, the global system of discretized equations describing the behavior of the analyzed continuum is:

$\mathbf{M} \ddot{\mathbf{U}}=\mathbf{F}^{\mathrm{ext}}-\mathbf{F}^{\mathrm{int}}-\mathbf{F}^{\mathrm{damp}}$

where $\mathbf{M}$ is the mass matrix, $\mathbf{U}$ is the vector of nodal displacements and $\mathbf{F}^{\text {int }}$ is the vector of internal nodal forces assembled as:

$\mathbf{F}^{\mathrm{int}}=\left.\sum_{I=1}^{N_{I P}}\left({ }_{0}^{t} \mathbf{F}_{0}^{t} \mathbf{B}_{L 0}^{T}{ }_{0}^{t} \hat{\mathbf{S}}\right)\right|_{\mathbf{x}=\mathbf{x}_{I}} W_{I}$

where, $N_{I P}$ is the total number of integration points (IP) distributed in the problem domain, ${ }_{0}^{t} \hat{\mathbf{S}}$ is the second Piola-Kirchoff stress vector at time $t,{ }_{0}^{t} \mathbf{F}$ is the deformation gradient between the undeformed configuration and the configuration at time $t, W_{I}$ is the weight corresponding to integration point $\mathbf{x}_{I},{ }^{0} \mathrm{~V}$ is the initial volume of the problem domain, and ${ }_{0}^{t} \mathbf{B}_{L 0}$ is the matrix of shape functions derivatives in reference to the initial configuration in the following form

$$
{ }_{0}^{t} \mathbf{B}_{L 0}=\left[\begin{array}{ccc}
\phi_{, x} & 0 & 0 \\
0 & \phi_{, y} & 0 \\
0 & 0 & \phi_{, z} \\
\phi_{, y} & \phi_{, x} & 0 \\
0 & \phi_{, z} & \phi_{, y} \\
\phi_{, z} & 0 & \phi_{, x}
\end{array}\right]
$$

in which $\phi_{, x}, \phi_{, y}$ and $\phi_{, z}$ are derivatives of shape functions with respect to $x, y$ and $z$, respectively.

As the purpose of this work is to verify the algorithm rather than to conduct a complete simulation of actual surgery, a simple Neo-Hookean hyperelastic model is a logical choice. The Neo-Hookean strain energy potential as used in ABAQUS is adopted in this work [27].

$W^{\text {Neo-Hooken }}=\frac{\mu_{0}}{2}\left(\bar{I}_{1}-3\right)+\frac{\kappa_{0}}{2}(J-1)^{2}$

where $\mu_{0}$ is the initial shear modulus, $\kappa_{0}$ is the initial bulk modulus, $J$ is the elastic volume ratio which equals to the determinant of deformation gradient, and $\bar{I}_{1}=J^{-\frac{2}{3}} \operatorname{tr}(\mathbf{C})$ is the first deviatoric strain invariant of the right Cauchy-Green deformation tensor $\mathbf{C}=\mathbf{F}^{T} \mathbf{F}$.

So the second Piola-Kirchoff stress in Eq. (6) is calculated as 
${ }_{0}^{t} \hat{\mathbf{S}}=\mu_{0} J^{-2 / 3}\left(\boldsymbol{\delta}-\frac{1}{3} \operatorname{tr}(\mathbf{C}) \mathbf{C}^{-1}\right)+\kappa_{0} J(J-1) \mathbf{C}^{-1}$

where $\boldsymbol{\delta}$ is the $3 \times 3$ identity matrix.

In Eq. (5) $\mathbf{F}^{\text {ext }}$ is the vector of externally applied forces (volumetric forces, surface and nodal forces) assembled as

$\mathbf{F}^{\mathrm{ext}}=\int_{\Omega} \boldsymbol{\Phi}_{I}^{T}(\mathbf{x}) \mathbf{b} \mathrm{d} \Omega+\int_{\Gamma} \boldsymbol{\Phi}_{I}^{T}(\mathbf{x}) \bar{\tau} \mathrm{d} \Gamma$

where $\mathbf{b}$ is the body force vector and $\bar{\tau}$ is the predefined surface traction on the boundary. The damping force $\mathbf{F}^{\text {damp }}=c \mathbf{M} \dot{\mathbf{U}}$, where $c$ is the damping coefficient, introduces a mass proportional damping which can be used to obtain the steady state solution, as presented in the next subsection.

\subsection{Dynamic Relaxation Solution Algorithm}

We introduce the damping force to dissipate the kinetic energy when that the steady state of the deformed continuum needs to be obtained, such damping does not have to be based on actual phenomena related to e.g. material viscosity. To increase the convergence speed towards the deformed state, we use the dynamic relaxation (DR) technique detailed in [28-30] and solve the damped equation of motion using the central difference method for integration in time domain. The resulting equation describing the iterations in terms of displacements, derived from Eq. (5), is:

$\mathbf{U}^{k+1}=\mathbf{U}^{k}+\beta\left(\mathbf{U}^{k}-\mathbf{U}^{k-1}\right)+\alpha \mathbf{M}^{-1}\left(\mathbf{F}^{\mathrm{ext}}-\mathbf{F}^{\mathrm{int}}\right)$

In the above equation $\alpha=2 h^{2} /(2+c h)$ and $\beta=(2-c h) /(2+c h)$, where $h$ is a fixed time increment and $k$ indicates the $k^{\text {th }}$ time increment. The iterative method defined by Eq. (11) is explicit as long as the mass matrix is diagonal. Therefore, we use a lumped mass matrix and a mass scaling algorithm that increases the convergence speed of the method. The accuracy of the solution is evaluated using a termination criterion which gives information about the absolute displacement error [28]:

$\left\|\mathbf{U}^{k+1}-\mathbf{U}^{*}\right\| \leq \frac{\omega}{1-\omega}\left\|\mathbf{U}^{k+1}-\mathbf{U}^{k}\right\| \leq \lambda$

where $\mathbf{U}^{*}$ is the solution, $\omega$ is the spectral radius which approximates the reduction in error, and $\lambda$ is the imposed accuracy. More details about the adaptive computation of these parameters in order to increase the convergence speed are given in [28]. 


\section{Numerical Implementation}

\subsection{Numerical Integration}

Compared to finite element method (FEM), which has well defined rules regarding numerical integration, accurate numerical integration for meshfree methods is still an open topic. Meshfree shape functions are generally not polynomials and are constructed using support nodes located beyond the boundaries of integration cells, which makes the numerical integration in meshfree methods much more challenging [31]. For meshfree methods, Gaussian quadrature over a background mesh is typically used for numerical integration [9, 20, 31]. Some efforts have been made on the numerical integration issue of meshfree methods, for example, the stabilized conforming nodal integration technique proposed by Chen et al. [32-34]. By developing the generalized gradient smoothing technique and using point interpolation method for field function approximation [35, 36], Liu et al. have developed a series of meshfree smoothed point interpolation methods (S-PIM) [37-40], which conduct stabilized numerical integrations on the generated smoothing domains.

In $[20,41]$ we used regular (equally spaced) hexahedral background grid to conduct the numerical integration in space. Such integration grid can be easily constructed, which offers simplicity and flexibility [20]. However, for complex geometries (such as those of many body organs) it may introduce volume inaccuracy when the hexahedra are intersected by a domain boundary. On possible solution to this problem can be to use tetrahedral background grids that can be automatically generated with integration cells conforming to the boundary of analyzed continuum even for complex geometries. Therefore, the meshfree algorithm we present in this study facilitates application of tetrahedral background integration grids.

For tetrahedral grid, we use four Gauss points per cell, which provides exact integration for polynomial up to $2^{\text {nd }}$ order. Unfortunately, the MLS shape functions are not polynomials and the Gaussian volumetric integration is not exact. Although there is no rigorous proof theory, some authors claimed [13] that the inaccuracy of numerical integration in meshfree methods can have similar effects to the reduced integration used in FEM to suppress volumetric locking for nearly incompressible materials. Although in FEM the reduced integration can lead to other numerical difficulties (hour-glassing), such problems can generally be avoided in meshfree methods by making influence domains larger. 


\subsection{Imposing Essential Boundary Conditions}

A critical feature of MLS shape functions is that they do not possess the Kronecker delta property (they are not interpolating). Consequently, essential boundary conditions cannot be imposed in the same way as in FEM. A number of techniques have been developed to overcome this problem, including the penalty method [42], the use of Lagrange multipliers in the Galerkin weak form [43] and the transform method [11].

In the explicit time integration framework, the most efficient and accurate method for imposing essential boundary conditions appears to be the coupling of MLS and FEM shape functions near the essential boundary [44]. Because the modifications are only made at shape functions level, the method can be easily applied to all kinds of problems and allows direct imposition of prescribed values to the field variables on the essential boundaries, just like in FEM. The coupling between FEM and MLS shape functions is accomplished by defining a boundary region that includes elements containing the nodes for which the essential boundary conditions are prescribed. The problem domain is divided into two regions, the boundary region $\Omega_{B}$ and the rest of the domain called the meshfree region $\Omega_{M}$. The displacement approximation in the boundary region is calculated as:

$$
u_{B}^{h}=\left\{\begin{array}{cc}
u^{M L S}+R\left(u^{F E M}-u^{M L S}\right) & \mathbf{x} \in \Omega_{B} \\
u^{M L S} & \mathbf{x} \in \Omega_{M}
\end{array}\right.
$$

where $u^{M L S}$ is the displacement field approximated using the MLS shape functions as in Eq. (3), $u^{F E M}$ is the displacement field approximated using the FEM shape functions, and $R$ is a ramp function [44].

\subsection{Stable Time Step Calculation}

We use explicit time integration in the present numerical algorithm because of its efficiency and suitability for parallel implementation. However explicit time integration is only conditionally stable and requires an estimation of the maximum stable time step. The critical time step of a numerical algorithm is directly related to the maximum frequency of free vibration, which is determined by the mass and stiffness of the system. Belytschko et al. developed critical time step bounds for meshfree methods, but their results are only valid for 2D problems with regularly distributed nodes [45]. Furthermore, the bounds become indefinite, due to division by zero, for interpolating shape functions or shape functions that are not strictly positive [46]. 
Recently Joldes et al. developed an effective method of estimating the stable time step for meshfree methods [47]. This estimation method is valid for a specific case of lumped mass matrix construction, where the mass associated with an integration point is distributed equally to the nodes influencing that integration point. This approach works well for the explicit time integration algorithm based on the EFG and is used in the present work for calculating the critical time step as

$$
\Delta t_{c r i t} \approx \min _{I}\left(\frac{2}{\sqrt{\lambda_{\max }^{I}}}\right)
$$

where $\lambda_{\max }^{I}$ is the maximum eigenvalue of the stiffness matrix contribution from integration point $I$, estimated as $\lambda_{\max }^{I} \leq n c_{w}{ }^{2} \cdot\left(\mathbf{B}_{L 0}\right)_{j I}^{I}\left(\mathbf{B}_{L 0}\right)_{j I}^{I}$, in which $n$ is the number of support nodes for integration point $I, C_{w}$ is the dilatational wave speed and $\mathbf{B}_{L 0}$ is the matrix of shape function derivatives. Following [48], the dilatational wave speed $c_{w}$ was calculated as $c_{w}=\sqrt{\frac{\lambda+2 \mu}{\rho}}$, where $\lambda$ and $\mu$ are Lamé’s constants.

\section{Numerical Verification}

A cylinder of height $0.1 \mathrm{~m}$ and radius $0.05 \mathrm{~m}$ is studied, as shown in Figure 1 . The cylinder is deformed by rigidly constraining one face $(z=0)$, while the opposite face $(z=0.1 \mathrm{~m})$ is displaced. We use the mass density of $1000 \mathrm{~kg} / \mathrm{m}^{3}$. As there is vast body of evidence [2, 49-60] that soft tissues can be regarded as hyperelastic (or hyperviscoelastic) nearly incompressible continuum, we used Neo-Hookean material with Young’s modulus in undeformed state equal to $3000 \mathrm{~Pa}$, Poisson's ratio of 0.49 , and density of $1000 \mathrm{~kg} / \mathrm{m}^{3}$. The loading (displacements) were applied using a smooth curve that ensures zero velocity and acceleration at the start of loading:

$d(t)=d_{\max } \cdot\left(10-15 t+6 t^{2}\right) \cdot t^{3}$ 


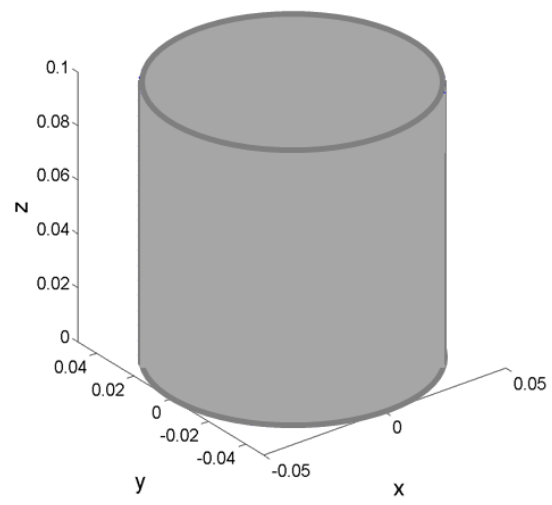

Figure 1. Thy cylinder is of height $0.1 \mathrm{~m}$ and radius $0.05 \mathrm{~m}$.

Three basic experiments are presented: compression, extension and shear of the cylinder. The computations were first performed using the proposed meshfree algorithm with a maximum applied displacement $d_{\max }=0.02 \mathrm{~m}$. The obtained results were compared to those from the commercial finite element software ABAQUS [27] using a fine mesh of 20-node quadratic brick, hybrid, linear pressure elements (C3D20H) and the dynamic implicit solver.

For the meshfree algorithm, the dilatation parameter for influence domain was taken as 1.6, the problem domain was discretized using 7,619 nodes and time step calculated using Eq. (14) is 8.2e-5 s. The reference solution of this problem was obtained with ABAQUS using a mesh consisting of 33,897 nodes and 7,680 elements. Table 1 lists the displacement in $x$ direction at the middle node of the right edge of plane $y=0$ and the total reaction force on the displaced face in the direction of the applied displacements (along the $\mathrm{z}$ axis for compression and tension, and along the $\mathrm{x}$ axis for shear). Compared with the reference solution, the maximum relative difference in both displacement and reaction force is smaller than $5 \%$, which conforms the accuracy of the results obtained using the presented algorithm. In Figure 2, we plotted the deformation shape of plane $y=0$, the displacements on the right edge of this plane and time history of the total reaction force on the displaced face. The results obtained using the presented meshfree algorithm closely agree with the reference solution from ABAQUS FE solver. 


\section{Table 1}

Displacement at the node $(0.05,0,0.05)$ in $x$ direction and reaction force on the displacement face.

\begin{tabular}{lllllll}
\hline & \multicolumn{2}{c}{ Compression } & \multicolumn{2}{c}{ Extension } & \multicolumn{2}{c}{ Shear } \\
\cline { 2 - 7 } & $\begin{array}{l}\text { Displacement } \\
(\mathrm{m})\end{array}$ & $\begin{array}{l}\text { Force } \\
(\mathrm{N})\end{array}$ & $\begin{array}{l}\text { Displacement } \\
(\mathrm{m})\end{array}$ & $\begin{array}{l}\text { Force } \\
(\mathrm{N})\end{array}$ & $\begin{array}{l}\text { Displacement } \\
(\mathrm{m})\end{array}$ & $\begin{array}{l}\text { Force } \\
(\mathrm{N})\end{array}$ \\
\hline Reference & 0.00703 & -6.9823 & -0.00508 & 4.6673 & 0.00951 & 1.1188 \\
Meshfree & 0.007315 & -7.2351 & -0.005144 & 4.7128 & 0.009614 & 1.1405 \\
Difference & $4 \%$ & $4 \%$ & $1 \%$ & $0.9 \%$ & $1 \%$ & $2 \%$ \\
\hline
\end{tabular}
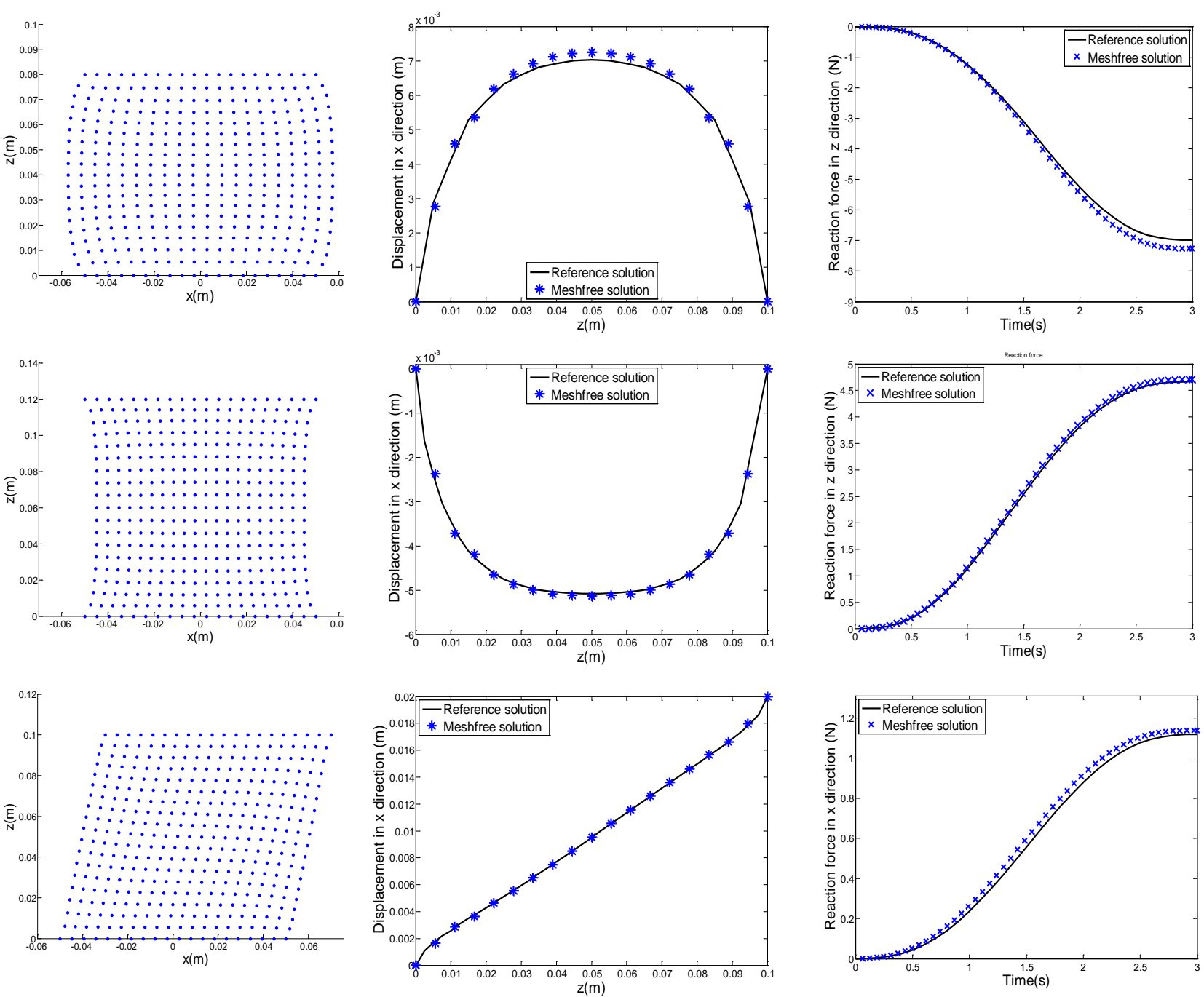

Figure 2. Compression, extension and simple shear of a cylinder. Left column: plane $y=0$ of the deformed cylinder. Middle column: displacements on the right edge of the plane $y=0$. Right column: time history of the total reaction force on the cylinder's displaced face. 


\section{Application in Soft Organ Modelling: Brain Indentation}

To illustrate the performance of the meshfree algorithm introduced in this study in simulation of the body organs we applied in modelling of the experiments on the swine brain indentation conducted by [61].

\subsection{Model Description}

The swine brain used in the experiment had a mass of $89.9 \mathrm{~g}$ and was approximately $92.5 \mathrm{~mm}$, $62.5 \mathrm{~mm}$ and $28.5 \mathrm{~mm}$ in its major axis, minor axis and height respectively. During the experiments the bottom brain surface was glued to a glass plate and the brain was laterally supported using two moulds (Figure 3a) with the brain surface firmly sticking to the moulds. Therefore, the nodes defining the bottom brain surface and the areas in contact with the moulds were rigidly constrained (Figure 3b). As in this study we focus on evaluation of the performance of our meshfree algorithm rather than on detailed modeling of the interactions between the indentor and brain, the indentor was not directly simulated in the model. The indentor was an aluminium cylinder of diameter 10 $\mathrm{mm}$. The loading was applied by prescribing a constant velocity of $1 \mathrm{~mm} / \mathrm{s}$ (velocity of the indetor) on the nodes located in the area that was in contact with the indentor when conducting the experiments.

Geometry for the brain model was obtained from the magnetic resonance image MRIs acquired before the experiments and discretized using 21,498 nodes and 115,029 tetrahedral integration cells as shown in Figure 3b. In the meshfree algorithm, the dilatation parameter for influence domain was taken as 1.6 and time step calculated using Eq. (14) is 4.3e-4 S. Neo-Hookean hyperelastic model was used for the brain tissue for verifying the developed algorithm. As explained in detail in [61], the subject-specific tissue properties (shear modulus of $210 \mathrm{~Pa}$ ) were determined from compression of the cylindrical tissue samples extracted from the brain after the indentation experiments. The Poisson's ratio was 0.49 and the mass density was $10^{3} \mathrm{~kg} / \mathrm{m}^{3}$. 


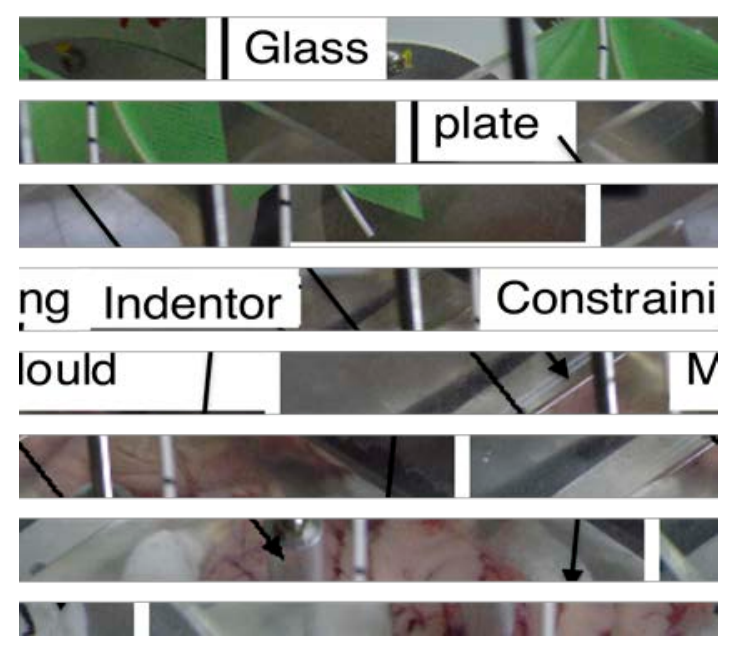

(a)

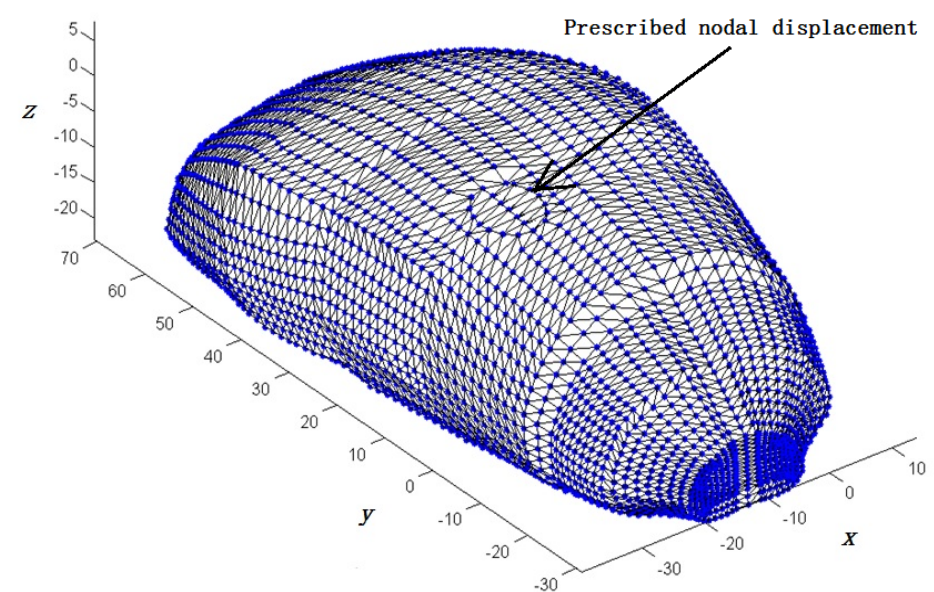

(b)

Figure 3. the swine brain indentation modelled using the presented meshfree algorithm: (a) Set-up of the experiments, Figure based on [61]; (b) domain discretization with scattered field nodes and tetrahedral integration grid, where the pointed circle area including the nodes under prescribed displacement during the indentation.

\subsection{Results}

As shown in Figure 4, the indentor reaction force-indentation depth relationship predicted by the model implemented using our meshfree algorithm agrees very well with experimental results throughout the simulation. These results also confirm that the algorithm facilitates robust and accurate solution even for large local deformation of the analyzed continuum.

The likely reasons for the differences between the simulation and experiment results in Figure 4 is that the pia mater (a think soft tissue layer surrounding the brain) was not included in the brain model we used (Figure 3b). As stated in Section 5.1, the purpose of this paper is to evaluate the performance of our meshree algorithm rather than to conduct a complete simulation of actual surgery. Given the fact that only very limited information about the mechanical properties of the pia mater is available [48] and that the anatomical structure of the soft tissue layers (meninges) surrounding the brain is still debated in the anatomical literature [62], attempt to include the pia mater in the model would obscure the algorithm evaluation with modeling uncertainties. 


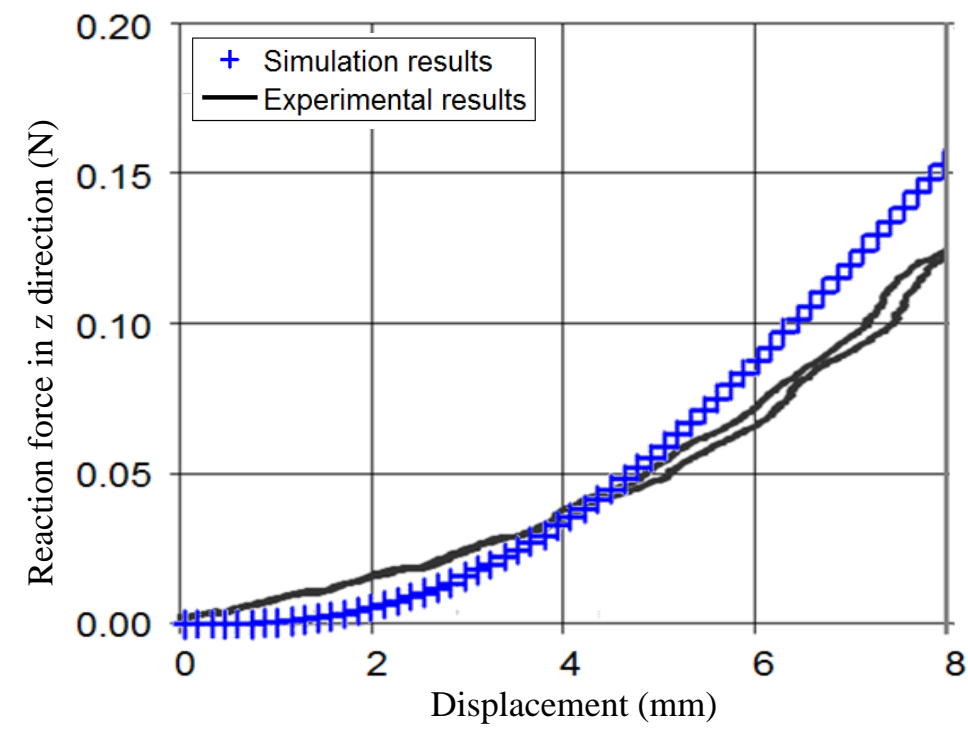

Figure 4. Comparison of the experimental and modeling results on the reaction force against displacement.

\section{Conclusions}

To address the need for simulating biological soft tissues under large deformations we presented a total Lagrangian explicit dynamics algorithm based on the element-free Galerkin method. The coupling between the moving least square (MLS) and finite element (FE) shape functions was used to impose essential boundary conditions, which is simple and guarantees exact results. The large support domains of the MLS shape functions eliminate some of the weaknesses of FE method, such as hour-glassing. Although the numerical volumetric integration is not exact, it seems to prevent volumetric locking for the studied nearly incompressible soft tissues materials. A dynamic relaxation scheme was used for the computation of the steady state solution and the critical time step estimation method ensures the stability of the present meshfree algorithm. The results of our computations compare very well with reference solutions obtained using the commercial FE software ABAQUS, confirming the accuracy of the presented algorithm. Application of the algorithm in modeling brain indentation indicates its ability to facilitate robust and accurate prediction of the organ responses.

\section{Acknowledgements}

The financial support of the Australian Research Council (Discovery grant No. DP1092893) and the University of Western Australia Research Development Awards 2012 is gratefully acknowledged. 


\section{References}

[1] Misra S, Ramesh KT, Okamura AM. Modeling of tool-tissue interactions for computer-based surgical simulation: A literature review. Presence-Teleoperators and Virtual Environments 2008;17(5): 463-91.

[2] Szekely G, Brechbuhler C, Hutter R, Rhomberg A, Ironmonger N, Schmid P. Modelling of soft tissue deformation for laparoscopic surgery simulation. Med Image Anal 2000;4: 57-66.

[3] Zhuang Y. Real-time Simulation of Physically Realistic Global Deformations [PhD Thesis]: University of California at Berkley; 2000.

[4] Miller K, Joldes G, Lance D, Wittek A. Total Lagrangian explicit dynamics finite element algorithm for computing soft tissue deformation. Commun Numer Meth En 2007;23(2): 121-34.

[5] Xu MH, Nowinski WL. Talairach-Tournoux brain atlas registration using a metalforming principle-based finite element method. Med Image Anal 2001;5(4): 271-79.

[6] Hu JW, Jin X, Lee JB, Zhang LZ, Chaudhary V, Guthikonda M, et al. Intraoperative brain shift prediction using a 3D inhomogeneous patient-specific finite element model. J Neurosurg 2007;106(1): 164-69.

[7] Wittek A, Joldes G, Couton M, Warfield SK, Miller K. Patient-specific non-linear finite element modelling for predicting soft organ deformation in real-time; Application to non-rigid neuroimage registration. Progress in Biophysics \& Molecular Biology 2010;103(2-3): 292-303.

[8] Belytschko T, Krongauz Y, Organ D, Fleming M, Krysl P. Meshless methods: An overview and recent developments. Computer Methods in Applied Mechanics and Engineering 1996;139: 3-47.

[9] Liu GR. Mesh Free Methods: Moving Beyond the Finite Element Method. Boca Raton: CRC Press; 2003.

[10] Li S, Liu WK. Meshfree Particle Methods: Springer-Verlag; 2004.

[11] Chen JS, Pan CH, Wu CT, Liu WK. Reproducing kernel particle methods for large deformation analysis of nonlinear structures. Computer Methods in Applied Mechanics and Engineering 1996;139(1-4): 195-227.

[12] Jun S, Liu WK, Belytschko T. Explicit reproducing kernel particle methods for large deformation problems. International Journal for Numerical Methods in Engineering 1998;41(1): 137-66.

[13] Li S, Hao W, Liu WK. Numerical simulations of large deformation of thin shell structures using meshfree methods. Computational Mechanics 2000;25(2-3): 102-16.

[14] Gu YT. Geometrically Nonlinear Analysis of Microswitches Using the Local Meshfree Method. International Journal of Computational Methods 2008;5(4): 513-32.

[15] Wang DD, Sun Y. A Galerkin Meshfree Method with Stabilized Conforming Nodal Integration for Geometrically Nonlinear Analysis of Shear Deformable Plates. International Journal of Computational Methods 2011;8(4): 685-703.

[16] Rabczuk T, Belytschko T. A three-dimensional large deformation meshfree method for arbitrary evolving cracks. Computer Methods in Applied Mechanics and Engineering 2007;196(29-30): 2777-99.

[17] Wang DD, Li ZY, Li L, Wu YC. Three dimensional efficient meshfree simulation of large deformation failure evolution in soil medium. Sci China Technol Sc 2011;54(3): 573-80.

[18] Gu YT, Wang QX, Lam KY. A meshless local Kriging method for large deformation analyses. Computer Methods in Applied Mechanics and Engineering 2007;196(9-12): 1673-84.

[19] Gu YT, Wang QX, Lam KY, Dai KY. A pseudo-elastic local meshless method for analysis of material nonlinear problems in solids. Engineering Analysis with Boundary Elements 2007;31(9): 771-82.

[20] Horton A, Wittek A, Joldes GR, Miller K. A meshless Total Lagrangian explicit dynamics algorithm for surgical simulation. International Journal for Numerical Methods in Biomedical Engineering 2010;26(8): 977-98.

[21] Doweidar MH, Calvo B, Alfaro I, Groenenboom P, Doblare M. A comparison of implicit and explicit natural element methods in large strains problems: Application to soft biological tissues modeling. Computer Methods in Applied Mechanics and Engineering 2010;199(25-28): 1691-700.

[22] De S, Lim YJ. Interactive Surgical Simulation Using a Meshfree Computational Method. Berlin: Springer-Verlag Berlin; 2010.

[23] Doblare M, Cueto E, Calvo B, Martinez MA, Garcia JM, Cegonino J. On the employ of meshless methods in biomechanics. Computer Methods in Applied Mechanics and Engineering 2005;194(6-8): 801-21.

[24] Ionescu I, Weiss JA, Guilkey J, Cole M, Kirby RM, Berzins M. Ballistic Injury Simulation Using the Material Point Method. In: Westwood JD, Haluck RS, Hoffman HM, Mogel GT, Phillips R, Robb RA, et al., editors. Medicine Meets Virtual Reality 14. Amsterdam: I O S Press; 2006. 228-33.

[25] Lancaster P, Salkauskas K. Surfaces Generated by Moving Least-Squares Methods. Math Comput 1981;37(155): $141-58$.

[26] Belytschko T, Lu YY, Gu L. Element-Free Galerkin Methods. International Journal for Numerical Methods in Engineering 1994;37(2): 229-56.

[27] ABAQUS. ABAQUS Theory Manual Version 5.2: Hibbit, Karlsson \& Sorensen, Inc.; 1998.

[28] Joldes GR, Wittek A, Miller K. An adaptive dynamic relaxation method for solving nonlinear finite element problems. Application to brain shift estimation. International Journal for Numerical Methods in Biomedical Engineering 2011;27(2): 173-85. 
[29] Sauve RG, Metzger DR. Advances in Dynamic Relaxation Techniques for Nonlinear Finite-Element Analysis. J Press Vess-T Asme 1995;117(2): 170-76.

[30] Joldes GR, Wittek A, Miller K. Computation of intra-operative brain shift using dynamic relaxation. Computer Methods in Applied Mechanics and Engineering 2009;198(41-44): 3313-20.

[31] Dolbow J, Belytschko T. Numerical integration of the Garlekin weak form in meshfree methods. Computational Mechanics 1999;23: 219-30.

[32] Chen JS, Wu CT, Yoon S, You Y. A stabilized conforming nodal integration for Galerkin mesh-free methods. International Journal for Numerical Methods in Engineering 2001;50(2): 435-66.

[33] Chen JS, Yoon SP, Wu CT. Non-linear version of stabilized conforming nodal integration for Galerkin mesh-free methods. International Journal for Numerical Methods in Engineering 2002;53(12): 2587-615.

[34] Wang DD, Lin ZT. Dispersion and transient analyses of Hermite reproducing kernel Galerkin meshfree method with sub-domain stabilized conforming integration for thin beam and plate structures. Computational Mechanics 2011;48(1): 47-63.

[35] Liu GR, Gu YT. A point interpolation method for two-dimensional solids. International Journal for Numerical Methods in Engineering 2001;50(4): 937-51.

[36] Liu GR, Gu YT, Dai KY. Assessment and applications of point interpolation methods for computational mechanics. International Journal for Numerical Methods in Engineering 2004;59(10): 1373-97.

[37] Liu GR, Zhang GY. Smoothed Point Interpolation Methods: G space and weakened weak forms. Singapore: World Scientific; 2013.

[38] Liu GR, Zhang GY, Dai KY, Wang YY, Zhong ZH, Li GY, et al. A linearly conforming point interpolation method (LC-PIM) for 2D solid mechanics problems. International Journal of Computational Methods 2005;2(4): 64565.

[39] Liu GR, Zhang GY. Edge-based smoothed point interpolation methods. International Journal of Computational Methods 2008;5(4): 621-46.

[40] Liu GR, Zhang GY. A Normed G Space and Weakened Weak (W(2)) Formulation of a Cell-Based Smoothed Point Interpolation Method. International Journal of Computational Methods 2009;6(1): 147-79.

[41] Jin X, Zhang G. Y., Joldes, G. R., Yang, K. H., Rohan, P. Y., Miller, K., Wittek, A. 2-D meshless algorithm for modelling of soft tissue undergoing fragmentation and large deformation: verification and performance evaluation. Proc. 10th International Symposium on Computer Methods in Biomechanics and Biomedical Engineering 2012.

[42] Atluri SN, Zhu T. A new meshless local Petrov-Galerkin (MLPG) approach in computational mechanics. Computational Mechanics 1998;22(2): 117-27.

[43] Belytschko T, Lu YY, Gu L. Element-free Galerkin methods. International Journal for Numerical Methods in Engineering 1994;37: pp. 229-56.

[44] Belytschko T, Organ D, Krongauz Y. A coupled finite element - Element-free Galerkin method. Computational Mechanics 1995;17(3): 186-95.

[45] Belytschko T, Guo Y, Liu WK, Xiao SP. A unified stability analysis of meshless particle methods. International Journal for Numerical Methods in Engineering 2000;48(9): 1359-400.

[46] Puso MA, Chen JS, Zywicz E, Elmer W. Meshfree and finite element nodal integration methods. International Journal for Numerical Methods in Engineering 2008;74(3): 416-46.

[47] Joldes GR, Wittek A, Miller K. Stable time step estimates for mesh-free particle methods. International Journal for Numerical Methods in Engineering 2012;91(4): 450-56.

[48] Belytschko T. An Overview of Semidiscretization and Time Integration Procedures. In: Belytschko T, Hughes TJR, editors. Computational Methods for Transient Analysis. Amsterdam: North-Holland; 1983. 1-66.

[49] Fung YC. Biomechanics. Mechanical Properties of Living Tissues. Second ed. New York: Springer-Verlag; 1993.

[50] Holbourn. Mechanics of head injury. The Lancet 1943;2: 438-4441.

[51] Pamidi MR, Advani SH. Nonlinear constitutive relations for human brain tissue. ASME Journal of Biomechanical Engineering 1978;100: 44-48.

[52] Walsh EK, Schettini A. Calculation of brain elastic parameters in vivo. American Journal of Physiology 1984;247: R637-R700.

[53] Farshad M, Barbezat M, Flüeler P, Schmidlin F, Graber P, Niederer P. Material characterization of the pig kidney in relation with the biomechanical analysis of renal trauma. J Biomech 1999;32(4): 417-25.

[54] Darvish K. Characterization of Non-Linear Viscoelastic Properties of Brain Tisse using Forced Vibrations [Doctoral dissertation]: University of Virginia; 2000.

[55] Mendis KK, Stalnaker RL, Advani SH. A constitutive relationship for large deformation finite element modeling of brain tissue. Journal of Biomechanical Engineering 1995;117: 279-85.

[56] Kauer M, Vuskovic V, Dual J, Szekely G, Bajka M. Inverse finite element characterization of soft tissues. Medical Image Analysis 2002;6(3): 275-86.

[57] Jin X, Lee JB, Leung LY, Zhang L, Yang KH, King AI. Biomechanical response of the bovine pia-arachnoid complex to tensile loading at varying strain-rates. Stapp Car Crash Journal 2006;50. 
[58] Jin X. Biomechanical response and constitutive modeling of bovine pia-arahcnoid complex [Ph.D.]. United States - Michigan: Wayne State University; 2009.

[59] Miller K, Chinzei K. Constitutive modelling of brain tissue; Experiment and Theory. Journal of Biomechanics 1997;30(11/12): 1115-21.

[60] Miller K. Constitutive modelling of abdominal organs. J Biomech 2000;33: 367-73.

[61] Wittek A, Dutta-Roy T, Taylor Z, Horton A, Washio T, Chinzei K, et al. Subject-specific non-linear biomechanical model of needle insertion into brain. Computer Methods in Biomechanics and Biomedical Engineering 2008;11(2): 135-46.

[62] Haines DE, Harkey HL, Al-Mefty O. The "subdural" space: A new look at an outdated concept. Neurosurgery 1993;32: 111-20. 